294 Eder: Verhalten von Leim u. Kohlehydraten etc. Ein zweiter Versuch gab:

$$
\mathrm{s}=2,945 \text { und } \mathrm{r}=113,8 \text {. }
$$

§39. Behandelt man ebenso das $\mathrm{K}_{2} \mathrm{CuS}_{2} \mathrm{O}_{8} .6 \mathrm{H}_{2} \mathrm{O}$, so erhält man abermals ein anderes Resultat. Wie ich schon in den "Dichtigkeitsmessungen" (1873) mitgetheilt habe, erleidet die geschmolzene und erstarrte Masse $\mathrm{K}_{2} \mathrm{Cu}_{2} \mathrm{O}_{8}$ während der Abkühlung eine Umwandlung, indem sie sich ausdehnt, unter Zerdrückung des Glasgefässes, in welchem sie geschmolzen wurde und erstarrt. Die Masse zerfällt dabei zu Pulver. Wäre sie eine Mischung von $\mathrm{K}_{2} \mathrm{SO}_{4}$ und $\mathrm{CuSO}_{4}$, so müsste sie weiss sein; wäre sie $\mathrm{K}_{2} \mathrm{SO}_{4} \cdot \mathrm{SO}_{3}$ und $\mathrm{CuO}$, so müsste sie grau bis schwarz sein; sie ist aber grün, glasig und homogen. Für das so erhaltene $\mathrm{K}_{2} \mathrm{Cu} \mathrm{S}_{2} \mathrm{O}_{8}$, $\mathrm{m}=337,4$ erhielt ich in $3 \mathrm{Wägungen:}$

$\mathrm{s}=1,754 ; \mathrm{s}=2,789$ und $\mathrm{s}=2,779$, entsprechend $\mathrm{v}=121,7$;

$$
\mathrm{v}=119,6 \text { und } \mathrm{v}=120,0 \text {. }
$$

Pettersson fand $\mathrm{v}=123,1$.

$\mathrm{Zu}$ einer Ansicht über die Volumconstitution dieser Verbindungen geben die Thatsachen noch keine genügende Grundlage.

Karlsruhe, den 7. März 1879.

\title{
Ueber das Verhalten von Leim und Kohlehydŗaten etc. gegen Chromate unter dem Einflusse des Lichtes;
}

von

\section{Dr. Josef Maria Eder.}

Es sind nun 40 Jahre verstrichen, seitdem Ponton zuerst beobachtete, dass Kaliumchromat durch organische Substanzen gebräunt wird; er machte diese Beobachtung, als er Papier mit Kaliumbichromat tränkte und dem Lichte aussetzte.

Seitdem wurden Gemenge von Kaliumbichromat mit mancherlei organischen Substanzen untersucht, so namentlich 
mit Gelatine, Albumin, Gummi Arabicum, Rohrzucker, Traubenzucker, Dextrin u. A. Alle diese Gemenge werden unter dem Einfluss des Lichtes in Wasser schwer- oder unlöslich. Auf diese Eigenschaft stützt sich ja die ganze moderne Chromatphotographie (Lichtdruck, Pigmentdruck, Photogalvanographie etc.). Ich studirte die diesbezüglichen Reactionen genauer.

Leim bildet mit Chromsäure ein Coagulum, welches sich beim Erhitzen löst. Nach dem Eintrocknen, auch bei gelinder Temperatur, wird das Gemenge in Wasser unlöslich, auch im Finstern; noch rascher geschieht dies im Tageslicht. Kaliumbichromat trocknet zu einer durchsichtigen Masse ein, welche das Salz derartig vertheilt enthält, dass keinerlei Krystallisation sichtbar wird. Erst bei grossen Mengen Chromat, etwa 20 Proc. vom Gewichte der Gelatine, krystallisirt es aus; gute Gelatinesorten vertragen mehr Salze als schlechte.

Im finsteren Raume oder bei inactinischem gelben Lichte aufbewahrt, verändert sich das getrocknete Gemenge von Leim mit Kalium- oder Ammoniumbichromat lange Zeit nicht. Es bleibt in heissem Wasser völlig löslich. Dagegen nach 3-14 Tagen - je nach der Temperatur der Luft und dem Feuchtigkeitsgehalte der Chromogelatine - wird das trockne Gemenge schwach bräunlich und ist in heissem Wasser unlöslich geworden. Bemerkenswerth ist, dass die Chromatgelatine bei erhöhter Temperatur und in feuchtem Zustande im Finstern am frühesten von selbst unlöslich wird. Ganz lufttrockne Chromatgelatineschichten bleiben viel länger unzersetzt, als gequollene, welche mitunter schon nach 24 Stunden ihre Löslichkeit in Wasser verloren haben. Erhitzt man trockne Chromatgelatine einige Stunden lang auf $100^{\circ}$, so wird sie in heissem Wasser schwer löslich, ja selbst bei 70 bis $80^{\circ}$ kann dieser Umstand eintreten. Am besten bewahren die bei 30 bis $40^{\circ}$ oder die im Exsiccator getrockneten Gemenge ihre Löslichkeit.

Gegen Licht (d. h. gegen blaues und violettes; rothes und gelbes ist nahezu ohne Wirkung) verhält sich das Ge- 
menge von Gelatine mit Bichromaten sehr verschieden, je nachdem es in wässriger Lösung oder in getrocknetem $\mathrm{Zu}$ stande belichtet worden. Lösungen zersetzen sich am Lichte sehr langsam. Ebenso sind anfgequollene Schichten sehr unempfindlich. Am empfindlichsten ist die lufttrockne Chromatgelatine, welche in Bruchtheilen einer Minute im Lichte die Löslichkeit im heissen Wasser verliert und gegen Licht viel empfindlicher als Chlorsilberpapier ist. Absolut wasserfreie, d. h. im Exsiccator über Schwefelsäure wohl getrocknete Chromatgelatine ist weniger leicht empfindlich als solche, welche aus der Luft hygroskopische Feuchtigkeit angezogen hat. Je mehr Chromsalze die Gelatine enthält, desto rascher wird sie im Lichte unlöslich. Chromatgelatine, welche eben beginnt, freiwillig im Dunkeln unlöslich zu worden, oder welche etwas wenig Chromalaun oder Tannin (also Substanzen, welche die Unlöslichkeit bedingen) enthalten, sind empfindlicher gegen Licht geworden.

Die Wirkung der Chromate auf den Leim ist variabel. Am lichtempfindlichsten sind Gremenge mit Kalium- und Ammoniumbichromat; ebenso verhält sich Ammoniummonochromat und Kaliumammoniumchromat, welche theils beim Trocknen, theils beim Belichten Ammoniak verlieren und in die entsprechenden Bichromate übergehen. Kaliummonochromat ist äusserst wenig empfindlich gegen Licht; das Gemenge mit Leim muss 20 bis $50 \mathrm{Mal}$ länger exponirt werden, um denselben Grad der Unlöslichkeit zu erlangen. Dagegen machen sämmtliche Monochromate den Iseim im Finstern viel weniger leicht unlöslich, als die Bichromate. Trichromate bewirken die Unlöslichkeit nach dem Trocknen auch bei gänzlichem Lichtabschluss.

Wie meine Analysen ergeben, ist die Wirkung des Lichtes auf ein Gemenge von Chromaten mit Leim ein einfacher Reductionsprocess. Die Chromsäure wird zu Chromoxyd reducirt, welche mit dem Bichromat sogenanntes chromsaures Chromoxyd bildet. Alle Chromoxydsalze aber machen den Leim in heissem Wasser unlöslich, namentlich wenn das Gemenge eingetrocknet war. Ich analysirte ein belichtetes Gemenge von Chromatgelatine, welches ich erhielt durch 
gegen Chromate unter dem Einflusse des Lichtes. 297

Aufquellen von Gelatine in einer gesättigten Kaliumbichromatlösung, Trocknen, tagelanges Belichten und Auskochen mit Wasser, bis dieses keine gelbe Färbung mehr annahm. Die Chromatgelatine $a$ und $b$ waren 2 und 3 Wochen, $c$ war nur 5 Tage exponirt. Ich fand:

\begin{tabular}{lrrr} 
& a. & b. & c. \\
Chromsüure & 7,03 & 5,93 & 1,84 \\
Chromoxyd & 15,68 & 12,47 & 3,36 \\
Aschenbestandtheile & 2,09 & 1,81 & 2,26 \\
Gelatine & 75,20 & 79,79 & 92,54 \\
\cline { 2 - 4 } & 100,00 & 100,00 & 100,00
\end{tabular}

Es ist also das chromsaure Chromoxyd ein integrirender Bestandtheil der belichteten Chromatgelatine. Dass das relative Verhältniss der Chromsäure und des Chromoxyds kein constantes ist, darf nicht auffallen; das chromsaure Chromoxyd ist ja eine sehr unbeständige Verbindung. Schon eine geringe Menge Chromoxyd genügt, um den Leim in kochendem Wasser unlöslich zu machen; als unterste Grenze ermittelte ich den Zusatz an 2 Proc. Chromalaun, welcher eben noch hinreicht.

Wie erwähnt, wird Chromatgelatine beim langen Liegen im Finstern oder beim Erhitzen ebenfalls unlöslich. Auch in derartigen Gemengen fand ich immer Chromoxyd.

Ein besonderes praktisches Interesse hat die Beantwortung der Frage, ob die belichtete Chromgelatine aus der Verbindung von chromsaurem Chromoxyd mit unveränderter Gelatine besteht, oder ob in dem Produlate die oxydirte Gelatine unlöslich als Hauptbestandtheil zurückbleibe.

Ein durch 14 Tage belichtetes trocknes Gemenge von Kaliumbichromat und Grelatine wurde mit Wasser, dann mit Ammoniak heiss digerirt und die gewaschene grüne Masse bei $120^{\circ}$ getrocknet und analysirt. Ich fand:

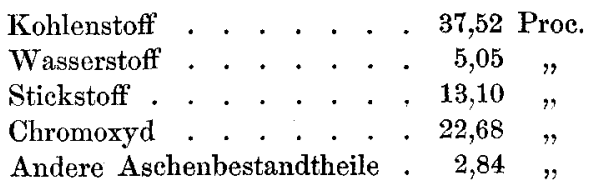


298 Eder: Verhalten von Leim u. Kohlehydraten etc.

Die Differenz ist Sauerstoff und eine geringe Menge von Schwefel. Wenn man nach Abzug der unorganischen Bestandtheile den Kohlenstoff, Wasserstoff und Stickstoff sich auf 100 Gewichtstheile der organischen Substanz berechnet, so erhält man folgende procentische Zusammensetzung der in der unlöslichen Chromatgelatine enthaltenen organischen Substanz.

\begin{tabular}{lrc} 
& \multicolumn{2}{c}{ Berechn. Zusammensetz. der } \\
& Gefunden. & Gelatine nach Muldex. \\
Kohlenstoff & 50,37 & 50,00 \\
Wasserstoff & 6,78 & 6,41 \\
Stickstoff & 17,59 & 17,95
\end{tabular}

Die im Lichte unlöslich gewordene Gelatine ist somit eine Verbindung von Chromoxyd mit unverändertem Leim. Die oxydirte organische Substanz, welche der Chromsäure den Sauerstoff entzog, befindet sich nicht im unlöslichen Theile. Ich suchte nun im löslichen Theile, indem ich 100 Grm. belichteter Chromatgelatine mit verdünnter Kalilauge auskochte, die Chromsäure im Filtrat mit Barytwasser fällte, filtrirte und nun mit Schwefelsäure destillirte. Das Destillat gab, mit Bleicarbonat abgestumpft, Krystalle von ameisensaurem Blei. Es waren also dieselben Oxydationsprodukte wie bei der Oxydation mit Kaliumbichromat und Schwefelsäure aufgetreten (neben Ameisensäure auch Essigsäure, Valeriansäure etc.).

Verhalten der belichteten unlöslichen Chromatgelatine (einer Verbindung von variablen Mengen Chromoxyd mit Leim) gegen Reagentien: Je länger die Belichtung gedauert hatte und je mehr Chromoxyd das Gemenge enthält, desto schwerer quillt sie in heissem Wasser auf; nur bei fortgesetztem Kochen löst sie sich auf. - Verdünnte Säuren und Alkalien bewirken die leichtere Auflösung in heissen Flüssigkeiten; in kurzer Zeit wird alle Chromsäure entzogen, dann lösen sich allmählich Chromoxyd und Leim gleichzeitig auf. - Ebenso bewirken heisse Lösungen Borax, Chlorwasser, Chlorkalk, Cyankalium eine beschleunigte Auflösung.

Zucker und Chromate sind im Dunkeln länger ohne Zersetzung haltbar als Leim. Rohrzuoker und Trauben- 
zucker verhalten sich ziemlich analog. Im Lichte sind ihre Gemenge mit Chromaten weniger zersetzlich als Leim; im trocknen Zustande sind sie ebenfalls empfindlicher als im gelösten. Ich setzte eine concentrirte Zuckerlösung mit Chromat während 3 bis 4 Wochen dem Lichte aus. In der stark gebräunten Masse suchte ich vergeblich nach Oxalsäure und Zuckersäure, welche sich in dem Produkte nach Swan und Krüger vorfinden sollen, sich aber nach meinen Versuchen nicht bilden. Dagegen konnte ich (nach der oben angegebenen Methode) Ameisensäure deutlich nachweisen. Das erbaltene Bleisalz hatte den Bleigehalt $(69,43$ bis 69,56 Proc.) des ameisensauren Salzes und reducirte Silber und Quecksilbersalze. Also im Licht geht auch hier dieselbe Oxydation vor sich, wie beim Erhitzen mit Chromat und Schwefelsäure, wobei ebenfalls das Hauptprodukt Ameisensäure ist.

Durch die Belichtung des Chromatzuckers büsst dieser seine Löslichkeit in Wasser nicht ein, dagegen verliert er seine hygroskopischen Eigenschaften, was namentlich beim Traubenzucker deutlich hervortritt.

Gummi arabicum wird durch Kaliumbichromat im Licht in kaltem und heissem Wasser unlöslich und verliert die hygroskopischen Eigenschaften. Auch bei diesem Körper sind die wässrigen Lösungen fast ganz unempfindlich gegen Lichtwirkungen. Um den Process zu studiren, stellte ich mir reine Arabinsäure und daraus gummisaures Kali dar, welches, bei $100^{\circ}$ getrocknet, 10,13 Proc. Kali enthielt. Diesen Körper mischte ich mit Bichromat; die nach mehrwöchentlicher Belichtung mit $W$ asser und Ammoniak behandelte und bei $120^{\circ}$ bis $130^{\circ}$ getrocknete Masse enthielt:

$\begin{array}{lrc}\text { Kohlenstoff } & 37,41 & \text { Proc. } \\ \text { Wasserstoff } & 5,63 & " \\ \text { Chromoxyd } & 5,76 & " \\ \text { Kali }\left(\mathrm{K}_{2} \mathrm{O}\right) & 9,30 \quad "\end{array}$

Der in dem Porzellanschiffchen verbleibende Rückstand enthielt Kaliumehromat und Carbonat nebst unzersetztem Chromoxyd, Der Inhalt wurde in ein Kölbchen gespült, 
300 Eder: Verhalten von Leim u. Kohlehydraten etc.

mit Salzsäure zerlegt und die entweichende Kohlensäure durch einen Kaliapparat geleitet, wie gewöhnlich bestimmt und zu der aus dem Verbrennungsrohre erhaltenen. Kohlensäure addirt. Das ungelöste Chromoxyd wurde auf einem Filter gesammelt, im Filtrat die Chromsäure zu Chromoxyd mit Alkohol reducirt und mit Ammoniak gefällt. Nachdem das Chromoxyd abfiltrirt war, wurde das Chlorkalium zur Trockne verdampft und nach dem gelinden Glühen als solches gewogen.

Die organische Substanz, nach Abzug der Mineralbestandtheile, lässt folgende Procentzusammensetzung berechnen:

\begin{tabular}{lcc} 
& \multicolumn{2}{c}{ Berechn. Zusammensetzung } \\
Kohlenstoff & Gefunden. & für $\mathrm{C}_{6} \mathrm{H}_{10} \mathrm{O}_{5}$. \\
Wasserstoff & 44,04 & 44,44 \\
& 6,63 & 6,17
\end{tabular}

Die Zusammensetzung der in dem unlöslichen belichteten Chromatgummi vorfindlichen organischen Substanz stimmt also sehr nahe mit der der Arabinsäure überein, und man kann annehmen, dass der unlösliche Bestandtheil aus gummisaurem (oder wahrscheinlicher metagummisaurem) Chromoxyd und Kali besteht. In der That fällt Chromalaun eine Gummilösung nicht, macht sie aber nach dem Eintrocknen in Wasser unlöslich.

Gummi Traganth verhält sich dem Gummi arabicum ähnlich.

Dextrin verhält sich im Gemenge mit Chromaten gegen das Licht ähnlicher dem Zucker, als dem Gummi arabicum. Er bräunt sich nämlich im Lichte, bildet chromsaures Chromoxyd und verliert die hygroskopischen Eigenschaften, ohne aber seine Löslichkeit in Wasser einzubüssen; erst nach dem wochenlangen Belichten tritt Unlöslichkeit ein.

Albumin ist mit Chromaten sehr lichtempfindlich; es wird am Lichte braun (chromsaures Chromoxyd) und in Wasser unlöslich, während das nicht belichtete eingetrocknete Gemenge von kaltem Wasser leicht gelöst wird. Das unlöslich gewordene Gemenge ist gegen Lösungsmittel widerstandsfähiger, als die anderen genannten Körper. Die wäss- 
rigen Gemische von Albumin nnd Chromaten halten sich im Dunkeln sehr lange unzersetzt (länger als Gelatine), namentlich wenn man überschüssiges Ammoniak zusetzt. Die Lichtwirkung auf ein Gemenge von Albumin und Kaliumbichromat kann man nicht ausschliesslich auf eine secundäre Wirkung des Chromates zurückführen, denn Chromoxyd bewirkt nicht die Unlöslichkeit des Albumins. Auch nach dem Eintrocknen bleiben Gemenge von Albumin und Chromalaun völlig löslich. Hier dürfte vielmehr eine höher zusammengesetzte Oxydationsstufe des Albumins (derlei Abkömmlinge giebt es ja viele) das Bild ausmachen, entweder in Verbindung mit Chromoxyd oder mit Albumin.

Glycerin reducirt allmählich im Lichte das Chromat und wird dann weniger hygroskopisch. Das Gemenge ist sehr unempfindlich.

Ein eingetrocknetes Gemenge von ammoniakalischer Caseïnlösung und Chromat giebt im Lichte ein Reactionsprodukt, welches in ammoniakalischem Wasser unlöslich ist, während es den nicht belichteten Theil löst.

Ammoniakalische Harzlösungen (Colophonium oder Schellack) mit Chromaten wurden weder in flüssiger, noch in trockner Form vom Lichte verändert. Es tritt keine Bräunung auf, noch werden die Gemenge in Ammoniak oder W eingeist unlöslich.

Weit ausführlicher als in dieser Skizze sind die erwähnten Reactionen in meinem von der Wiener photographischen Gesellschaft preisgekrönten Werkchen: „Ueber die Reactionen der Chromsäure und der Chromate gegen Gelatine, Zucker etc. in ihren Beziehungen zur Chromatphotographie" (Wien 1878) beschrieben und die Quellen mit genauen Literaturnachweisen angeführt.

Troppau, Staats-Oberrealschule. 